Pacific Journal of Mathematic 


\title{
OPERATORS INVERTIBLE MODULO THE WEAKLY COMPACT OPERATORS
}

\author{
KUNG-WEI YANG
}

\begin{abstract}
A continuous linear operator is a Fredholm operator if and only if it is invertible modulo the compact operators. In this note, we will generalize several theorems on Fredholm operators to theorems concerning operators invertible modulo the weakly compact operators.
\end{abstract}

1. Preliminaries. We fix the following notation.

$C=$ the complex field

$B=$ the category of complex Banach spaces and continuous linear operators

$B(X, Y)=$ the Banach space of continuous linear operators from $X$ to $Y$ (with the sup norm ||)

$W K(X, Y)=$ the closed subspace of all weakly compact operators in $B(X, Y)$

$X^{*}=B(X, C)$, the conjugate space

$F^{*}=B(F, C)$, the adjoint of $F: X \rightarrow Y$

$I_{X}=$ the identity operator on $X$

$\bar{X}=X^{* *} / n_{X}(X)$, where $n_{X}: X \rightarrow X^{* *}$ is the natural injection.

If $F \in B(X, Y)$, then the commutative diagram with exact rows

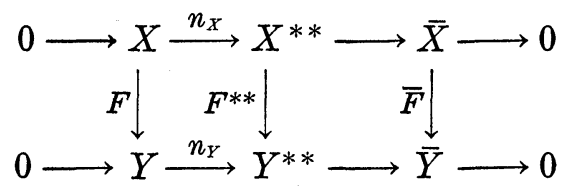

uniquely defines an operator $\bar{F} \in B(\bar{X}, \bar{Y})$. (Here $n_{X}, n_{Y}$ are the natural injections.)

We will need the following results (1.1)-(1.7) from [9].

1.1. $X$ is reflexive if and only if $\bar{X}=0 . \quad[9,(3.1)]$

1.2. $F \in W K(X, Y)$ if and only if $\bar{F}=0$. [9, (4.1)]

1.3. $\bar{I}_{X}=I_{\bar{X}}$. $[9,(2.3)]$

1.4. If $E \in B(X, Y)$ and $F \in B(Y, Z)$, then $\overline{F E}=\overline{F E}$. [9, (2.3)]

1.5. $|\bar{F}| \leqq|F|$. $\quad[9,(2.3)]$

1.6. For any $a, b \in C$ and $E, F \in B(X, Y), \overline{a E+b F}=a \bar{E}+b \bar{F}$. $[9,(2.4)]$

1.7. There exists a natural topological isomorphism $N_{X}:(\bar{X})^{*} \rightarrow$ $\left(\bar{X}^{*}\right)$. i.e., given any $F \in B(X, Y)$, the diagram 


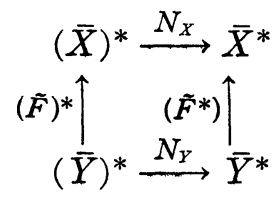

is commutative. $[9,(2.8)]$ (The naturality is not explicitly stated in [9].)

THEOREM 1.8. If $E \in B\left(X, X_{1}\right)$ and $F \in B\left(Y, Y_{1}\right)$, then $\overline{E \oplus F}=$ $\bar{E} \oplus \bar{F}$, where $\oplus$ denotes direct sum.

Proof. Use $(E \oplus F)^{* *}=E^{* *} \oplus F^{* *}$ and the following commutative diagram with exact rows:

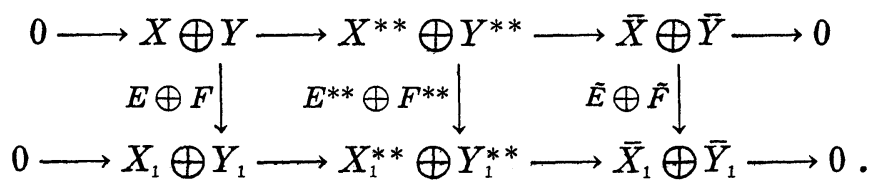

2. The operators invertible modulo the weakly compact operators. An operator $F \in B(X, Y)$ is left (right) invertible modulo the weakly compact operators if there exists an operator $E \in B(Y, X)$ such that $\overline{E F}=I_{\bar{X}}\left(\overline{F E}=I_{\bar{Y}}\right)$. An operator is invertible modulo the weakly compact operators if it is left and right invertible modulo the weakly compact operators. Notice that this condition is quite different from merely requiring $\bar{F}$ to be invertible. We let $\Psi_{l}(X, Y)$, $\Psi_{r}(X, Y)$, denote the set of all operators left, respectively right, invertible modulo the weakly compact operators and let $\Psi(X, Y)$ denote the set of all operators invertible modulo the weakly compact operators.

THEOREM 2.1. If $E \in \Psi(X, Y)$ and $F \in \Psi(Y, Z)$. Then $F E \in$ $\Psi(X, Z)$.

Proof. By assumption there exist $E_{1} \in B(Y, X), F_{1} \in B(Z, Y)$ such that $\overline{E_{1} E}=I_{\bar{X}}, \overline{E E}_{1}=I_{\bar{Y}}, \overline{F_{1} F}=I_{\bar{X}}, \overline{F F_{1}}=I_{Z}$. Clearly,

$$
\left(\overline{E_{1} F_{1}}\right)(\overline{F E})=I_{\bar{X}}
$$

and $\left.\overline{(F E)\left(E_{1} F_{1}\right.}\right)=I_{\bar{Z}}$.

THEOREM 2.2. Let $E \in B(X, Y), F \in B(Y, Z)$. Assume $F E \epsilon$ $\Psi(X, Z)$. Then,

(1) $E \in(X, Y)$ if and only if $F \in \Psi(Y, Z)$;

(2) If $F \in \Psi_{l}(Y, Z)$, then $E \in \Psi(X, Y)$ and $F \in \Psi(Y, Z)$;

(3) If $E \in \Psi_{r}(X, Y)$, then $E \in \Psi(X, Y)$ and $F \in \Psi(Y, Z)$. 
Proof. By assumption, there exist $G \in B(Z, X)$ such that $\overline{G F E}=$ $I_{\bar{X}}$ and $\overline{F E G}=I_{\bar{Z}}$.

(1) If $E \in \Psi(X, Y)$, then there exists $E_{1} \in B(Y, X)$ such that $\overline{E_{1} E}=I_{\bar{X}}$ and $\overline{E E_{1}}=I_{\bar{Y}}$. Hence $\overline{E G F}=I_{\bar{Y}}$ and $\overline{F(E G)}=I_{\bar{Z}}$. This means $F \in \Psi(Y, Z)$. The implication in the other direction is proved similarly.

(2) If $F \in \Psi_{l}(Y, Z)$, then there exists $F_{1} \in B(Z, Y)$ such that $\overline{F_{1} F}=I_{\bar{Y}}$. This clearly implies $\overline{(G F) E}=I_{\bar{X}}, \overline{E(G \bar{F})}=I_{\bar{Y}}$ and $\overline{=F(E G)}$ $I_{\bar{Z}},\left(\overline{E G) F}=I_{\bar{Y}}\right.$. Hence, $E \in \Psi(X, Y)$ and $F \in \Psi(Y, Z)$.

(3) is proved similarly.

Theorem 2.3. Let $F \in B(X, Y)$. If there exist $E_{1}, E_{2} \in B(Y, X)$ such that $E_{1} F$ and $F E_{2}$ are invertible modulo the weakly compact operators, then $F \in \Psi(X, Y)$.

Proof. Since $E_{1} F$ and $F E_{2}$ are invertible modulo the weakly compact operators, there exist $G_{1} \in B(X, X), G_{2} \in B(Y, Y)$ such that $\overline{G_{1}\left(E_{1} F\right)}=I_{\bar{X}}$ and $\overline{\left(F E_{2}\right) G_{2}}=I_{\bar{Y}}$. Hence $F \in \Psi(X, Y)$.

Theorem 2.4. If $F \in \Psi(X, Y)$, then $F^{*} \in \Psi\left(Y^{*}, X^{*}\right)$.

Proof. Let $F \in \Psi(X, Y)$. Then there exists $E \in B(Y, X)$ such that $\overline{E F}=I_{\bar{X}}$ and $\overline{F E}=I_{\bar{Y}}$. By 1.7, $\overline{E^{*} F^{*}}=\left(N_{Y}(\bar{E})^{*} N_{\bar{X}}^{-1}\right)\left(N_{X}(\bar{F})^{*} N_{\bar{Y}}^{-1}\right)=$ $I_{\bar{Y}^{*}}$ and $\overline{F^{*} E^{*}}=\left(N_{X}(\bar{F})^{*} N_{Y}^{-1}\right)\left(N_{Y}(\bar{E})^{*} N_{X}^{-1}\right)=I_{\bar{X}^{*}}$. Hence $F^{*} \in \Psi\left(Y^{*}, X^{*}\right)$.

Theorem 2.5. If $F \in \Psi(X, Y)$ and $K \in W K(X, Y)$, then $F+K \in$ $\Psi(X, Y)$.

\section{Proof. $\overline{F+K}=\bar{F}+\bar{K}=\bar{F}$.}

As is shown in [9, Theorem 5.10], if the Banach spaces $X$ and $Y$ enjoy the property that every closed reflexive subspace of $X$ is complemented and every closed subspace of $Y$ with reflexive quotient is complemented, then every generalized Fredholm operator is invertible modulo the weakly compact operators.

There are, however, other kinds of operators invertible modulo the weakly compact operators. Let $X$ be any Banach space. Let $U: X \rightarrow X$ be an invertible operator, and $K \in W K(X, X)$. Then, clearly, $U+K \in \Psi(X, X)$.

To construct a nontrivial operator invertible modulo the weakly compact operators which is not a generalized Fredholm operator, we start an operator $F=U+K \in \Psi(X, X)$ such as the one constructed above. We choose a reflexive Banach space $Y$ and an operator $G \epsilon$ $B(Y, Y)$ which does not have a closed range. If we form the direct 
sum $F \oplus G: X \oplus Y \rightarrow X \oplus Y$, we see, by Theorem 1.8, $\overline{F \oplus G}=$ $\bar{F} \oplus \bar{G}=\bar{F} \oplus 0$. Hence $F \oplus G$ is invertible modulo the weakly compact operators but it is definitely not a generalized Fredholm operator because it does not have a closed range. (Also see [3, V. 2.6].)

3. The operators left (right) invertible modulo the weakly compact operators.

Theorem 3.1. (1) If $F \in \Psi_{l}(X, Y), K \in W K(X, Y)$, then $F+K \in$ $\Psi_{l}(X, Y)$.

(2) If $E \in \Psi_{l}(X, Y), F \in \Psi_{l}(Y, Z)$, then $F E \in \Psi_{l}(X, Z)$.

(3) If $E \in B(X, Y), F \in B(Y, Z)$ and $F E \in \Psi_{\imath}(X, Z)$, then $E \in$ $\Psi_{l}(X, Y)$.

(4) If $F \in \Psi_{r}(X, Y), K \in W K(X, Y)$, then $F+K \in \Psi_{r}(X, Y)$.

(5) If $E \in \Psi_{r}(X, Y), F \in \Psi_{r}(Y, Z)$, then $F E \in \Psi_{r}(X, Z)$.

(6) If $E \in B(X, Y), F \in B(Y, Z)$ and $F E \in \Psi_{r}(X, Z)$, then $F \in$ $\Psi_{r}(Y, Z)$.

Proof. (1) $\overline{F+K}=\bar{F}+\bar{K}=\bar{F}$.

(2) If $E \in \Psi_{l}(X, Y)$ and $F \in \Psi_{l}(Y, Z)$, then there exist $E_{1} \in$ $B(Y, X), F_{1} \in B(Z, Y)$ such that $\overline{E_{1} E}=I_{\bar{X}}$ and $\overline{F_{1} F}=I_{\bar{Y}}$. Clearly, $\overline{E_{1} F_{1} F E}=I_{\bar{X}}$. Hence $F E \in \Psi_{l}(X, Z)$.

(3) If $F E \in \Psi_{l}(X, Z)$, then there exists $G \in B(Z, X)$ such that $\overline{G(F E)}=I_{\bar{X}}$. Hence $E \in \Psi_{l}(X, Y)$.

(4), (5), (6) are similarly proved.

THEOREM 3.2. (1) If $F \in \Psi_{l}(X, Y)$, then $F^{*} \in \Psi_{r}\left(Y^{*}, X^{*}\right)$.

(2) If $F \in \Psi_{r}(X, Y)$, then $F^{*} \in \Psi_{l}\left(Y^{*}, X^{*}\right)$.

Proof. (1) If $F \in \Psi_{l}(X, Y)$, then there exists $F_{1} \in B(Y, X)$ such that $\overline{F_{1} F}=I_{\bar{X}}$. Hence $(\bar{F})^{*}\left(\bar{F}_{1}\right)^{*}=I_{(\bar{X}) * \bullet}$ By 1.7,

$$
\left(N_{X}(\bar{F}) * N_{Y}^{-1}\right)\left(N_{Y}\left(\bar{F}_{1}\right)^{*} N_{X}^{-1}\right)=I_{\bar{X}^{*}},
$$

whence $\overline{F^{*} F_{1}^{*}}=I_{X^{*}}$. This shows $F^{*} \in \Psi_{r}\left(Y^{*}, X^{*}\right)$.

(2) is proved similarly.

4. Perturbation. Let $A$ be a Banach algebra with identity (1), $A^{\circ}$ be the group of invertible elements in $A, A_{l}^{\circ}\left(A_{r}^{\circ}\right)$ be the set of all left(right) invertible elements of $A$. Let

$R(A)=$ the radical of $A$

$=\left\{r \in A \mid 1+a r \in A^{\circ}\right.$ for every $\left.a \in A\right\}[6$, p. 163]

$=\left\{r \in A \mid 1+a r \in A^{\circ}\right.$ for every $\left.a \in A^{\circ}\right\}[5, \mathrm{p} .4]$ 
$Q(A)=$ the set of all quasi-nilpotent (topologically nilpotent [7, p. 12]) elements of $A$

$=\left\{q \in A \mid 1+k q \in A^{\circ}\right.$ for every $\left.k \in C\right\}[4$, p. 699]

$=\left\{\left.q \in A|| q^{n}\right|^{1 / n} \rightarrow 0\right.$ as $\left.n \rightarrow \infty\right\}$. [1, p. 23]

For a semigroup $S$ in $A$, let

$$
P(S)=\{a \in A \mid a+S \subset S\} .
$$

The following theorem is proved in [5].

TheOREM 4.1. $P\left(A^{\circ}\right)=P\left(A_{l}^{\circ}\right)=P\left(A_{r}^{\circ}\right)=R(A)$.

THEOREM 4.2 .

$Q(A)=\left\{q \in A \mid I+a q \in A^{\circ}\right.$ for all $a \in A^{\circ}$ such that $\left.a q=q \alpha\right\}$.

Proof. The set on the right is obviously contained in $Q(A)$. Now take an element $q \in Q(A)$, and let $a \in A^{\circ}$ be such that $\alpha q=q a$. Clearly, $\left|(\alpha q)^{n}\right|^{1 / n} \rightarrow 0$ as $n \rightarrow \infty$. Hence $a q \in Q(A)$. So $1+a q \in A^{\circ}$. This shows that $q$ is in the set on the right hand side.

TheOREm 4.3. (1) Let $q \in A$. Then, $q \in Q(A)$ if and only if for all $a \in A^{\circ}$ such that $a q=q a, a+q \in A^{\circ}$;

(2) If $q_{1}, q_{2} \in Q(A)$ and $q_{1} q_{2}=q_{2} q_{1}$, then $q_{1}+q_{2} \in Q(A)$.

Proof. (1) Clearly, $a q=q a$ is equivalent to $a^{-1} q=q a^{-1}$. Hence, $q \in Q(A) \Leftrightarrow 1+a q \in A^{\circ}$ for all $a \in A^{\circ}$ such that $a q=q a \Leftrightarrow 1+a^{-1} q \epsilon$ $A^{\circ}$ for all $a \in A^{\circ}$ such that $a q=q a \Leftrightarrow a+q=a\left(1+a^{-1} q\right) \in A^{\circ}$ for all $a$ such that $a q=q a$.

(2) Let $q_{1}, q_{2} \in Q(A)$ be such that $q_{1} q_{2}=q_{2} q_{1}$. Let $k$ be an arbitrary complex number. Clearly, $\left(1+k q_{1}\right) \in A^{\circ}$ and $k q_{2} \in Q(A)$. Since $\left(1+k q_{1}\right)\left(k q_{2}\right)=\left(k q_{2}\right)\left(1+k q_{2}\right)$, by $(1), 1+k\left(q_{1}+q_{2}\right) \in A^{\circ}$. Since $k$ is arbitrary, $q_{1}+q_{2} \in Q(A)$.

We remark that (2) also follows from [7, Th. 1.4.1(v), p. 10].

Now we shall apply these theorems to the specific problem of perturbation of operators (left, right) invertible modulo the compact operators.

Let $X$ be a Banach space. Let $B(X)=B(X, X), W K(X)=$ $W K(X, X), \Psi(X)=\Psi(X, X), \Psi_{l}(X)=\Psi_{l}(X, X), \Psi_{r}(X)=\Psi_{r}(X, X)$, $\bar{B}(X)=B(X) / W K(X)$ and $\tau: B(X) \rightarrow \bar{B}(X)$ be the natural projection. Notice that $\bar{B}(X)$ can be considered as a subalgebra of $B(\bar{X})$ [9, (5.11)] and we have $\Psi(X)=\tau^{-1}\left[(\bar{B}(X))^{\circ}\right],{ }_{l} \psi(X)=\tau^{-1}\left[(\bar{B}(X))_{l}^{\circ}\right], \Psi_{r}(X)=$ $\tau^{-1}\left[(\bar{B}(X))_{r}^{\circ}\right]$.

THEOREM 4.4. $P(\Psi(X))=P\left(\Psi_{l}(X)\right)=P\left(\Psi_{r}(X)\right)=\tau^{-1}[R(\bar{B}(X))]$. 
Proof. Use Theorem 4.1 and the above remark.

CoROLlaRY 4.5. If $R(\bar{B}(X))=0$, then $P(\Psi(X))=P\left(\Psi_{l}(X)\right)=$ $P\left(\Psi_{r}(X)\right)=W K(X)$.

Since $\bar{B}(X)$ can be considered as a subalgebra of $B(\bar{X})$ and since $R(B(\bar{X}))=0$ [4, p. 702], $R(\bar{B}(X))=0$ if $\bar{B}(X)=B(\bar{X})$.

Now, let $\Omega(X)=\tau^{-1}[Q(\bar{B}(X))]$. The classical counterpart of $\Omega(X)$ is the set of all Riesz operators [2, p. 323] [8].

THEOREM 4.6. Let $E \in B(X)$. Then, $E \in \Omega(X)$ if and only if $E+F \in \Psi(X)$ for all $F \in \Psi(X)$ such that $\overline{E F}=\overline{F E}$.

Proof. Apply Theorem 4.3.1.

THEOREM 4.7. If $E_{1}, E_{2} \in \Omega(X)$ and ${\overline{E_{1}}}_{2}={\overline{E_{2}}}_{1}$, then $E_{1}+E_{2} \in$ $\Omega(X)$.

\section{Proof. Apply Theorem 4.3.2.}

Note that if we apply Theorem 4.3 to the case $A=B(X) / K(X)$, where $K(X)$ is the closed 2-sided ideal of all compact operators, then we obtain Theorems $9,12,13$, of [8]. They are the classical "relatives" of Theorems 4.6 and 4.7 .

It would be an interesting problem to characterize the operators in $\Psi(X, Y)\left(\Psi_{t}(X, Y), \Psi_{r}(X, Y)\right)$ intrinsically.

We would like to thank Professor Martin Schechter for his help.

\section{REFERENCES}

1. F. F. Bonsall and J. Duncan, Complete Normed Algebras, Springer-Verlag, New York, 1973.

2. J. Dieudonné, Foundations of Modern Analysis, Academic Press, New York, 1967.

3. S. Goldberg, Unbounded Linear Operators, McGraw-Hill, New York, 1966.

4. E. Hille and R. S. Phillips, Functional analysis and semigroups, Amer. Math. Soc. Colloq. Publ., 1957.

5. A. Lebow and M. Schechter, Semigroups of operators and measures of noncompactness, J. Functional Analysis, 7 (1971), 1-26.

6. M. A. Naimark, Normed Rings, P. Noordhoff, N. V. Groningen, 1959.

7. C. E. Rickart, General Theory of Banach Algebras, V. Nostrand, Princeton, N. J., 1960.

8. M. Schechter, Riesz operators and Fredholm perturbations, Bull. Amer. Math. Soc., 74 (1968), 1139-1144.

9. K.-W. Yang, The generalized Fredholm operators, Trans. Amer. Math. Soc., (to appear).

Received February 11, 1976 and in revised form February 4, 1977.

WeSTERN Michigan UNIVERSITY

KaLAMAZO0, MI 49008 


\section{PACIFIC JOURNAL OF MATHEMATICS}

\section{EDITORS}

RICHARD ARENS (Managing Editor)

University of California

Los Angeles, CA 90024

Charles W. Curtis

University of Oregon

Eugene, OR 97403

C. C. MOORE

University of California

Berkeley, CA 94720

\section{J. DugundJI}

Department of Mathematics

University of Southern California

Los Angeles, CA 90007

R. FinN and J. Milgram

Stanford University

Stanford, CA 94305

ASSOCIATE EDITORS
E. F. BECKENBACH
B. H. NeumanN
F. WOLF
K. YOSHIDA

\section{SUPPORTING INSTITUTIONS}

UNIVERSITY OF BRITISH COLUMBIA CALIFORNIA INSTITUTE OF TECHNOLOGY

UNIVERSITY OF CALIFORNIA

MONTANA STATE UNIVERSITY

UNIVERSITY OF NEVADA, RENO

NEW MEXICO STATE UNIVERSITY

OREGON STATE UNIVERSITY

UNIVERSITY OF OREGON

OSAKA UNIVERSITY

\author{
UNIVERSITY OF SOUTHERN CALIFORNIA \\ STANFORD UNIVERSITY \\ UNIVERSITY OF HAWAII \\ UNIVERSITY OF TOKYO \\ UNIVERSITY OF UTAH \\ WASHINGTON STATE UNIVERSITY \\ UNIVERSITY OF WASHINGTON \\ AMERICAN MATHEMATICAL SOCIETY
}

The Supporting Institutions listed above contribute to the cost of publication of this Journal, but they are not owners or publishers and have no responsibility for its content or policies.

Mathematical papers intended for publication in the Pacific Jaurnal of Mathematics should be in typed form or offset-reproduced, (not dittoed), double spaced with large margins. Please do not use built up fractions in the text of your manuscript. You may however, use them in the displayed equations. Underline Greek letters in red, German in green, and script in blue. The first paragraph or two must be capable of being used separately as a synopsis of the entire paper. Items of the bibliography should not be cited there unless absolutely necessary, in which case they must be identified by author and Journal, rather than by item number. Manuscripts, in triplicate, may be sent to any one of the editors. Please classify according to the scheme of Math. Reviews, Index to Vol. 39. All other communications should be addressed to the managing editor, or Elaine Barth, University of California, Los Angeles, California, 90024.

The Pacific Journal of Mathematics expects the author's institution to pay page charges, and reserves the right to delay publication for nonpayment of charges in case of financial emergency.

100 reprints are provided free for each article, only if page charges have been substantially paid. Additional copies may be obtained at cost in multiples of 50 .

The Pacific Journal of Mathematics is issued monthly as of January 1966. Regular subscription rate: $\$ 7200$ a year (6 Vols., 12 issues). Special rate: $\$ 36.00$ a year to individual members of supporting institutions.

Subscriptions, orders for back numbers, and changes of address should be sent to Pacific Journal of Mathematics, 103 Highland Boulevard, Berkeley, California, 94708.

PUBLISHED BY PACIFIC JOURNAL OF MATHEMATICS, A NON-PROFIT CORPORATION

Printed at Kokusai Bunken Insatsusha (International Academic Printing Co., Ltd.). 8-8, 3-chome, Takadanobaba, Shinjuku-ku, Tokyo 160, Japan.

Copyright (C) 1975 by Pacific Journal of Mathematics Manufactured and first issued in Japan 


\section{Pacific Journal of Mathematics}

\section{Vol. 71, No. $2 \quad$ December, 1977}

Krishnaswami Alladi and Paul Erdős, On an additive arithmetic

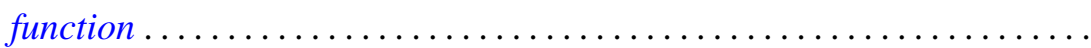

James Bailey and Dale Rolfsen, An unexpected surgery construction of a

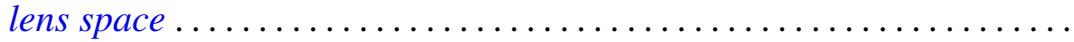

Lawrence James Brenton, On the Riemann-Roch equation for singular

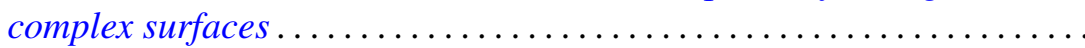

James Glenn Brookshear, Projective ideals in rings of continuous

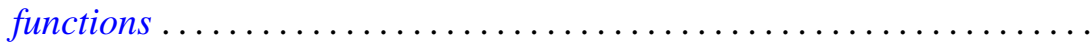

Lawrence Gerald Brown, Stable isomorphism of hereditary subalgebras of

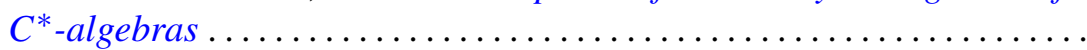

Lawrence Gerald Brown, Philip Palmer Green and Marc Aristide Rieffel, Stable isomorphism and strong Morita equivalence of $C^{*}$-algebras....

N. Burgoyne, Robert L. Griess, Jr. and Richard Lyons, Maximal subgroups

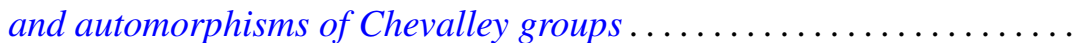
365

Yuen-Kwok Chan, Constructive foundations of potential theory .... 405

Peter Fletcher and William Lindgren, On $w \Delta$-spaces, $w \sigma$-spaces and

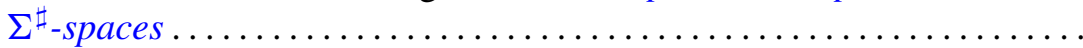

Louis M. Friedler and Dix Hayes Pettey, Inverse limits and mappings of

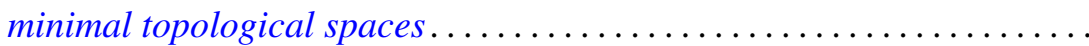

Robert E. Hartwig and Jiang Luh, A note on the group structure of unit regular ring elements.

I. Martin (Irving) Isaacs, Real representations of groups with a single involution ...

Nicolas P. Jewell, The existence of discontinuous module derivations . .

Antonio M. Lopez, The maximal right quotient semigroup of a strong semilattice of semigroups .......................

Dennis McGavran, $T^{n}$-actions on simply connected $(n+2)$-manifolds

Charles Anthony Micchelli and Allan Pinkus, Total positivity and the exact $n$-width of certain sets in $L^{1}$.

Barada K. Ray and Billy E. Rhoades, Fixed point-theorems for mappings with a contractive iterate .......................

Fred Richman and Elbert A. Walker, Ext in pre-Abelian categories. .

Raymond Craig Roan, Weak* generators of $H^{\infty}$ and $l^{1}$..

Saburou Saitoh, The exact Bergman kernel and the kernels of Szegö type...

Kung-Wei Yang, Operators invertible modulo the weakly compact 